\title{
Network Meta-Analysis of Metabolic Surgery Procedures for the Treatment of Obesity and Diabetes
}

\author{
Andrew C. Currie ${ }^{1} \cdot$ Alan Askari $^{2} \cdot$ Ana Fangueiro $^{2} \cdot$ Kamal Mahawar $^{3}$ \\ Received: 22 February 2021 / Revised: 30 July 2021 / Accepted: 30 July 2021 / Published online: 7 August 2021 \\ (c) The Author(s), under exclusive licence to Springer Science+Business Media, LLC, part of Springer Nature 2021
}

\begin{abstract}
Background Metabolic surgery is part of a well-established treatment intensification strategy for obesity and its related comorbidities including type 2 diabetes (T2DM). Roux-en-Y gastric bypass (RYGB), sleeve gastrectomy (SG) and oneanastomosis gastric bypass (OAGB) are the most commonly performed metabolic surgeries worldwide, but comparative efficacy is uncertain. This study employed network meta-analysis to compare weight loss, T2DM remission and perioperative complications in adults between RYGB, SG and OAGB.

Methods MEDLINE, EMBASE, trial registries were searched for randomised trials comparing RYGB, SG and OAGB. Study outcomes were excess weight loss (at 1,2 and 3-5 years), trial-defined T2DM remission at any time point and perioperative complications.

Results Twenty randomised controlled trials were included involving 1803 patients investigating the three metabolic surgical interventions. RYGB was the index for comparison. The excess weight loss (EWL) demonstrated minor differences at 1 and 2 years, but no differences between interventions at 3-5 years. T2DM remission was more likely to occur with either RYGB or OAGB when compared to SG. Perioperative complications were higher with RYGB when compared to either SG or OAGB. Two-way analysis of EWL and T2DM remission against the risk of perioperative complications demonstrated OAGB was the most positive on this assessment at all time points.

Conclusion OAGB offers comparable metabolic control through weight loss and T2DM remission to RYGB and SG whilst minimising perioperative complications.
\end{abstract}

Registration number: CRD42020199779 (https:// www.crd.york.ac.uk/PROSPERO)

Keywords Metabolic surgery · Obesity · Type 2 diabetes $\cdot$ Network meta-analysis

\section{Introduction}

Key Points

- Excess weight loss similar for RYGB, SG and OAGB

- T2DM remission more likely with RYGB or OAGB

- Perioperative complications more common with RYGB

- RYGB, SG or OAGB should be considered as primary metabolic surgical procedures

Andrew C. Currie

andrew.currie@nhs.net

Alan Askari

alan.askari@gmail.com

Ana Fangueiro

ana.macielfangueiro@study.beds.ac.uk

Kamal Mahawar

kmahawar@gmail.com
Metabolic surgery improves mortality and morbidity outcomes in patients with obesity [1] and has been shown to be cost-effective $[2,3]$. A Cochrane systematic review found metabolic surgery was more effective than non-surgical obesity treatment [4]. Data from the Swedish Obese Subjects Study found a $25 \%$ weight loss at 10 years in those

1 Department of Bariatric Surgery, Ashford \& St Peter's Hospital NHS Trust, Guildford Street, Chertsey KT16 0PZ, UK

2 Department of Bariatric Surgery, Luton and Dunstable NHS Trust, Luton, UK

3 Bariatric Unit, Sunderland Royal Hospital, Sunderland, UK 
undergoing gastric bypass surgery with a $24 \%$ decrease in mortality compared with the control group [1]. Metabolic surgery improves functional impairment and cardiovascular disease and reduces cancer risk and mortality related to type 2 diabetes (T2DM) [5-8]. Furthermore, metabolic surgery has been shown in a number of trials to induce remission of T2DM and has been endorsed by 200 diabetes organisations worldwide as a standard of care [9]. People living with obesity have been shown to have higher intensive care admissions and mortality during the recent coronavirus pandemic $[10,11]$ which has brought focus to the delivery of metabolic surgical services [12, 13]. However, studies demonstrate only a small proportion of the eligible population receive surgical intervention for obesity-related metabolic disease [14, 15].

Metabolic surgery is part of a well-established treatment intensification strategy for obesity-related comorbidities [16, 17], but the role of individual operative approaches is less certain. The Fourth Global International Federation for the Surgery of Obesity (IFSO) Registry in 2018 reported that of the 190,177 primary metabolic operations, sleeve gastrectomy (SG) was the most common $(87,467 ; 46.0 \%)$ followed by Roux-en-Y gastric bypass (RYGB) $(72,645 ; 38.2 \%)$ and one-anastomosis gastric bypass (OAGB) $(14,516 ; 7.6 \%)$ [18]. Previously published standard pairwise meta-analyses have produced inconsistent findings [19-22]. With the evolution of procedures and the increasing use and understanding of one-anastomosis gastric bypass [23], an updated comparative efficacy assessment is required to aid healthcare decision making.

This study employed network meta-analysis (NMA) to compare weight loss, type 2 diabetes remission and perioperative complications in adults between Roux-en-Y gastric bypass, sleeve gastrectomy and one-anastomosis gastric bypass.

\section{Methods}

A systematic review was undertaken to identify all published randomised controlled trials (RCTs) evaluating Roux-en-Y gastric bypass, sleeve gastrectomy and one-anastomosis gastric bypass. The review was conducted in line with the PRISMA statement [24] and PRISMA-Network meta-analysis extension [25]. The review was registered in the PROSPERO database (CRD42020199779).

\section{Search Strategy}

Medline, Embase, the Cochrane Register of Clinical Trials and the International Standard Randomised Controlled Trial Number (ISRCTN) Registry (controlled-trials.com) were searched from January 2000 until August 2020 using a search strategy based on excess weight loss, remission of type 2 diabetes and perioperative complications for (1) Roux-en-Y gastric bypass (RYGB), (2) sleeve gastrectomy (SG) or (3) one-anastomosis gastric bypass (OAGB) (Appendix). Randomised controlled trials evaluating at least two of the techniques in adult patients for the treatment of obesity or related comorbidities were included. Trials recruiting only children and those not reporting any of the primary outcomes of interest were excluded. No language restrictions were employed. Abstracts and conference proceedings were not included as they provide insufficient detail. Two authors trained in systematic review techniques (ACC \& AA) completed the search strategy and screening, with disagreements resolved through consensus of the wider study team.

\section{Outcome Measures}

All RCTS including the primary outcomes of interest, including percentage excess weight loss, remission of diabetes (trialist-defined) and perioperative complications (trialist-defined), were included.

\section{Data Extraction}

Arm level data were extracted from the included studies, from communication with authors or from previous metaanalyses where the original study authors had been contacted for data verification. After a calibration exercise, three authors (ACC, AA \& AF) independently performed data extraction. Disagreement at all stages was resolved by discussion with the senior author (KM).

\section{Quality Assessment}

Potential biases were identified using the Cochrane risk of bias tool [26]: sequence generation, allocation concealment, blinding, if the outcomes reported were prespecified, completeness of outcome data and other potential sources of bias. Three authors (ACC, AA \& AF) independently performed the quality assessment. Disagreement was resolved by discussion with the wider study team.

\section{Statistical Methods}

Random-effects network meta-analysis using the frequentist approach was applied to synthesis evidence for the primary outcomes. Evidence was summarised in a network map for each outcome. A common within network estimate for heterogeneity was estimated with the restricted maximum likelihood method [27, 28]. Consistency between direct and indirect evidence or between studies involving different sets of treatments for the same comparison was tested using the 
design by treatment interaction model [29]. Inconsistency was assessed using the node-splitting method [30, 31].

Where outcomes demonstrated statistically significant differences between procedures, the ranking probabilities of each metabolic surgical procedure (RYGB was the reference) were calculated and presented as rankograms. The ranking (surface under the cumulative ranking curve, SUCRA) scores for each treatment for the most commonly reported effectiveness (percentage excess weight loss and remission of diabetes) and adverse (perioperative complication) outcomes were then combined by time point into clustered ranking plots, to enable a simultaneous comparison of benefits and risks. Sensitivity analyses were undertaken excluding trials where the risk of bias was considered to be high due to poor allocation concealment or randomisation processes.

The assessment of statistical heterogeneity in the entire network was based on the magnitude of the heterogeneity variance parameter $(\tau)$ estimated from the NMA models, which is the estimated standard deviation treatment effects. We applied a 0.5 zero-cell correction before metaanalysis. To investigate potential small-study effects, a comparison-adjusted funnel plot was constructed. Statistical analysis was performed in STATA 16.0 (StataCorp, College Station, TX, USA) using 'mvmeta' and 'network' commands and other routines as reported [32-34]. All statistical tests were two sided, with the threshold of significance set at a $p$ value of less than 0.05 .

\section{Role of the Funding Source}

There was no funding source for this study. All authors had full access to all the data in the study and had final responsibility for the decision to submit for publication.

\section{Results}

Following systematic searches in MEDLINE, EMBASE and trial registries, 2291 abstracts were identified, and following exclusions, 25 articles regarding 20 RCTs (1803 patients) were included [35-59] (Fig. 1). The characteristics of the included trials are shown in Table 1 and the risk-of-bias assessment shown in Fig. 2. The most commonly studied
Fig. 1 PRISMA diagram for study inclusion

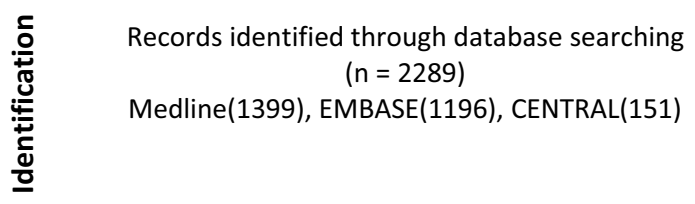

Records after duplicates removed

$$
(n=1811)
$$

ํㅗำ

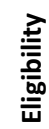

$\frac{7}{ㅇ ㅗ ㅇ ~}$
Studies included in qualitative synthesis

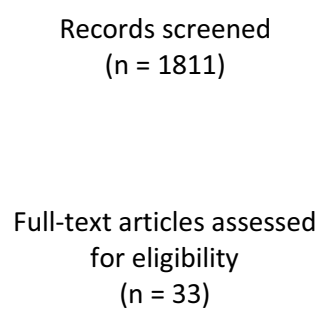
Full-text articles assessed for eligibility

$(n=33)$

Studies included in quantitative synthesis (meta-analysis) $(n=20)$
Additional records identified from systematic reviews $(n=4)$
Records excluded ( $n=1778$ )

Full-text articles excluded $(n=13)$

Non-included metabolic or bariatric surgery trial arm $(n=11)$

No primary outcome data $(n=2)$ 


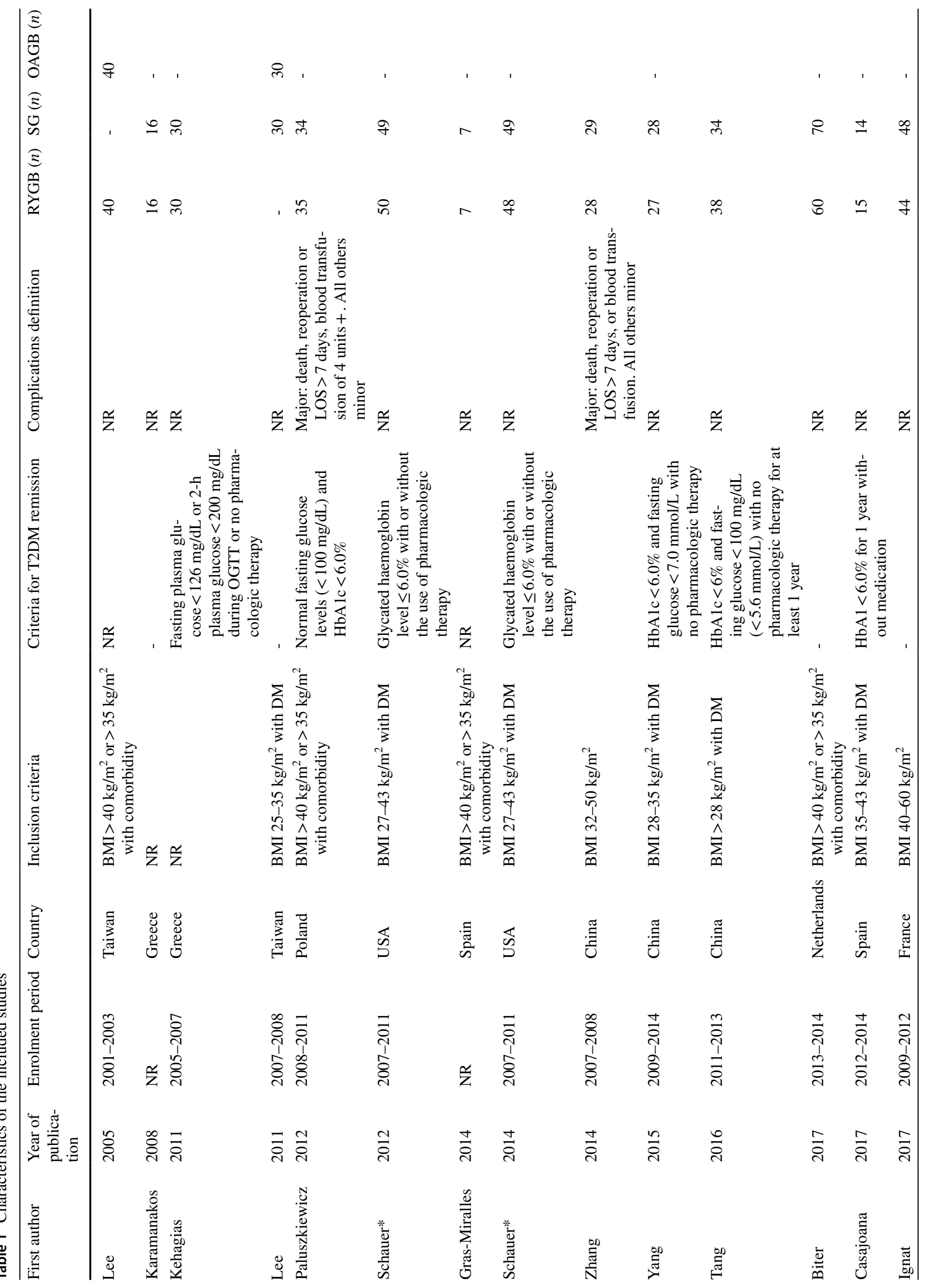




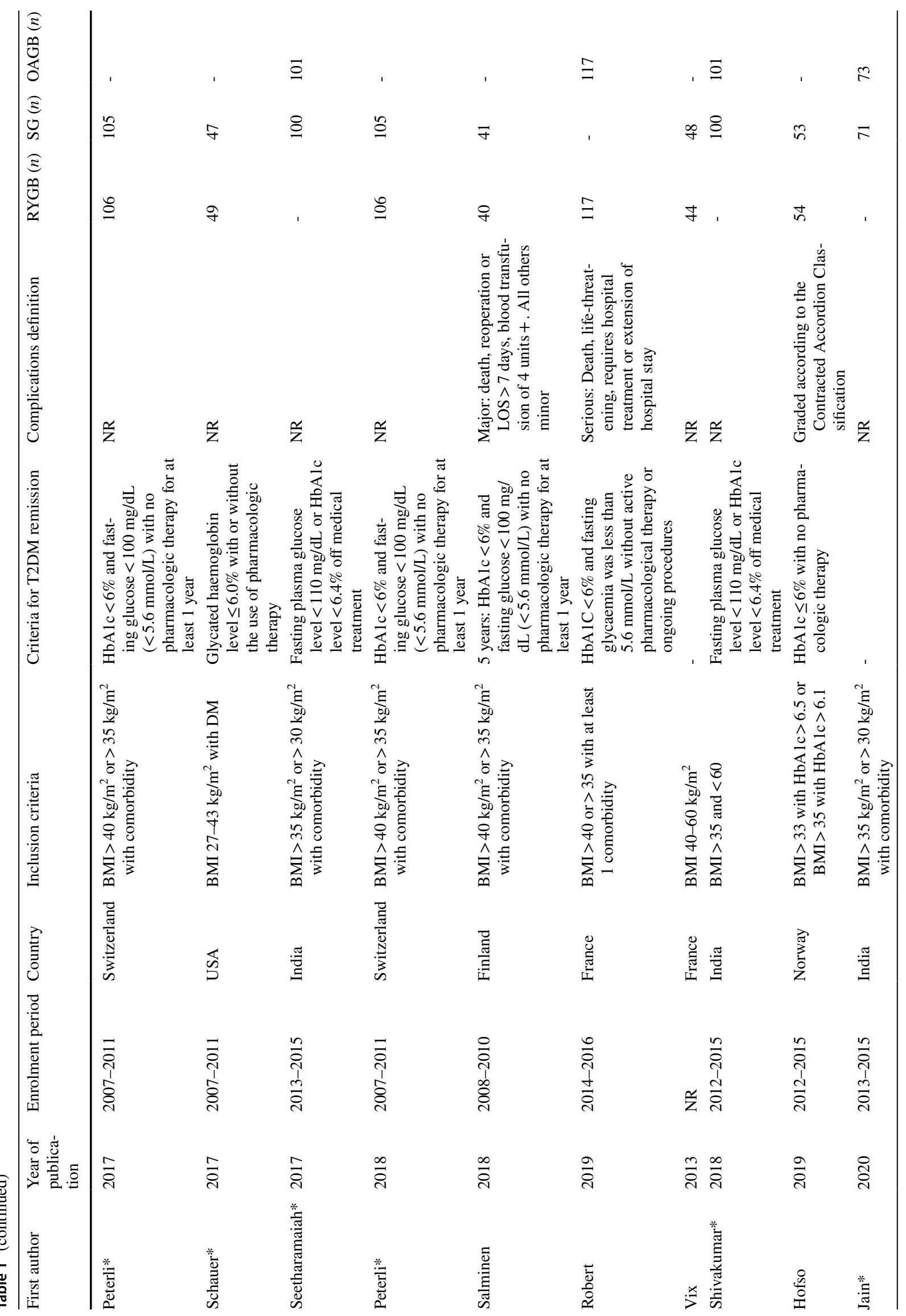


randomised comparison was between RYGB and SG (13 RCTs) for excess weight loss (EWL) (13 RCTs) and T2DM remission (12 RCTs) (Fig. 3). Whilst the overall risk of bias was low in the included articles, $8 / 25$ had an unclear risk of bias related the randomisation process, and 4/25 had an unclear risk of bias regarding outcome reporting. No evidence of small-study effects on the network were identified on the comparison-adjusted funnel plot (Fig. 4).

\section{Excess Weight Loss}

At 1 year, 13 trials including 1270 randomised patients were included in the comparison of percentage excess weight loss [35, 37, 39-44, 46, 52, 54, 57, 58] (Table 2). For 1-year EWL, 8 RCTs compared RYGB with SG, 2 RCTs compared RYGB v OAGB and 3 RCTS compared SG with OAGB. In standard meta-analysis, no approach was favoured over another for EWL at 1 year. On network meta-analysis, RYGB and SG had similar EWL at 1 year, but OAGB had greater EWL at 1 year than both RYGB and SG. In the NMA, there was no inconsistency seen between the direct and network effect sizes $\left(\chi^{2}=8.56,3\right.$ d.f., $\left.P=0.0357\right)$. No evidence of statistical heterogeneity was seen in the network $\left(\tau^{2}<0.001\right)$.

At 2 years, 4 RCTs compared RYGB with SG, 2 RCTs compared RYGB v OAGB and 2 RCTs compared SG with OAGB [39, 41, 43, 46, 47, 53, 57, 59] (Table 2). In standard meta-analysis, no approach was favoured over another for EWL at 2-year follow-up. On network meta-analysis, RYGB and SG had similar EWL at 2 years, whereas OAGB has slightly greater EWL at 2 years when compared to RYGB. SG and OAGB had similar EWL at 2 years. In the NMA, there was no inconsistency seen between the direct and network effect sizes $\left(\chi^{2}=9.50,3\right.$ d.f., $\left.P=0.0187\right)$. No evidence of statistical heterogeneity was seen in the network $\left(\tau^{2}<0.001\right)$.

At 3-5 years, 7 RCTs compared RYGB with SG, 1 RCT compared RYGB v OAGB and 2 RCTS compared SG with OAGB [39, 41, 46, 48, 55-59] (Table 2). In standard metaanalysis, RYGB had greater EWL than SG at between 3 and 5 years postoperatively. No differences in EWL at this time point were noted in standard meta-analysis of the two trials of SG and OAGB. On network meta-analysis, RYGB had greater EWL than SG at 3-5 years, whereas RYGB and OAGB had similar EWL at this time point. SG and OAGB had similar EWL at 3-5 years. In the NMA, there was no inconsistency seen between the direct and network effect sizes $\left(\chi^{2}=10.16,3\right.$ d.f., $\left.P=0.0257\right)$. No evidence of statistical heterogeneity was seen in the network $\left(\tau^{2}<0.001\right)$.

\section{Diabetes Remission}

Fourteen trials including 697 randomised patients with T2DM were included in the comparison of diabetes 
Fig. 2 Cochrane risk of bias-2 tool assessments of quality of included studies
Risk of bias domains

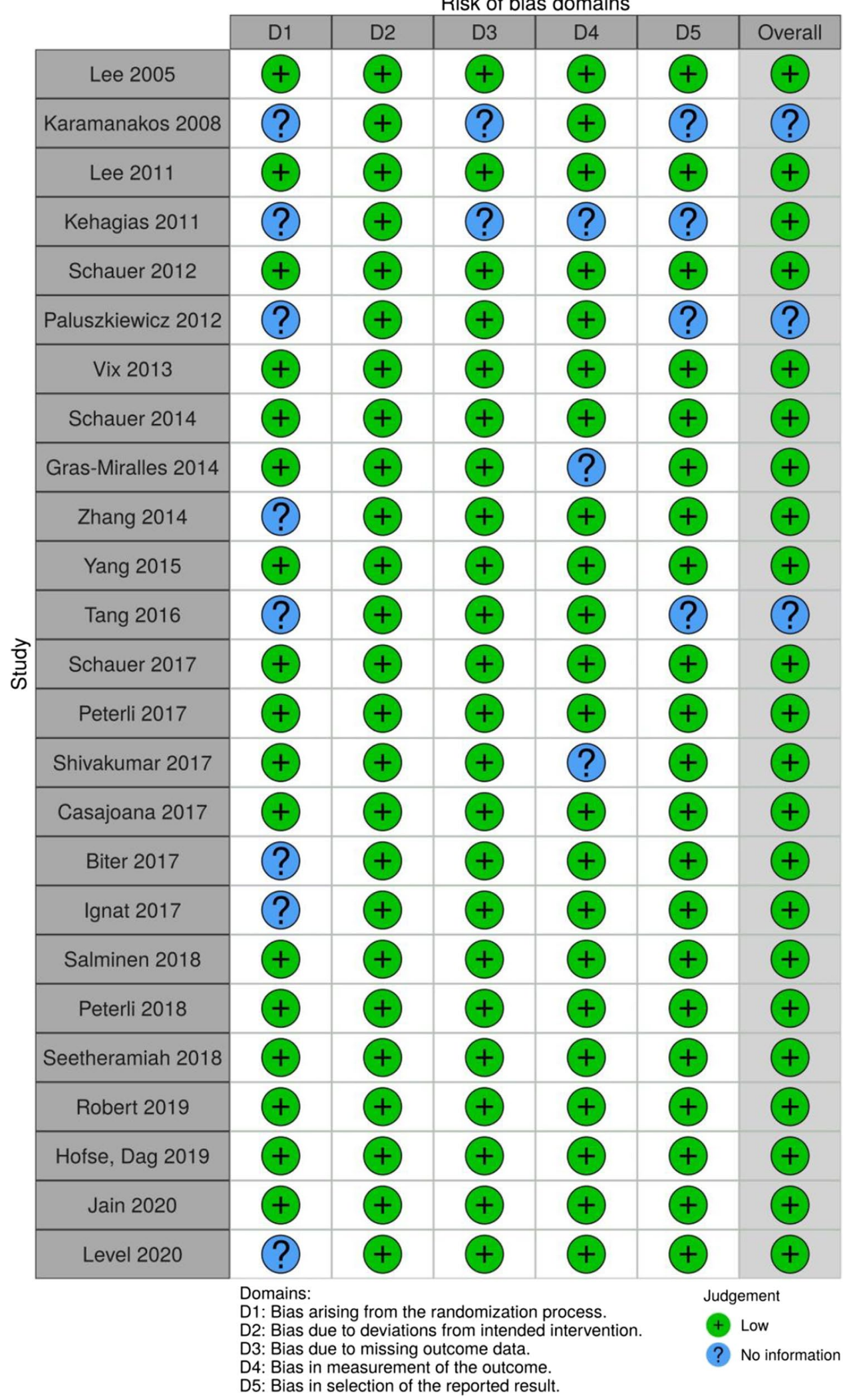



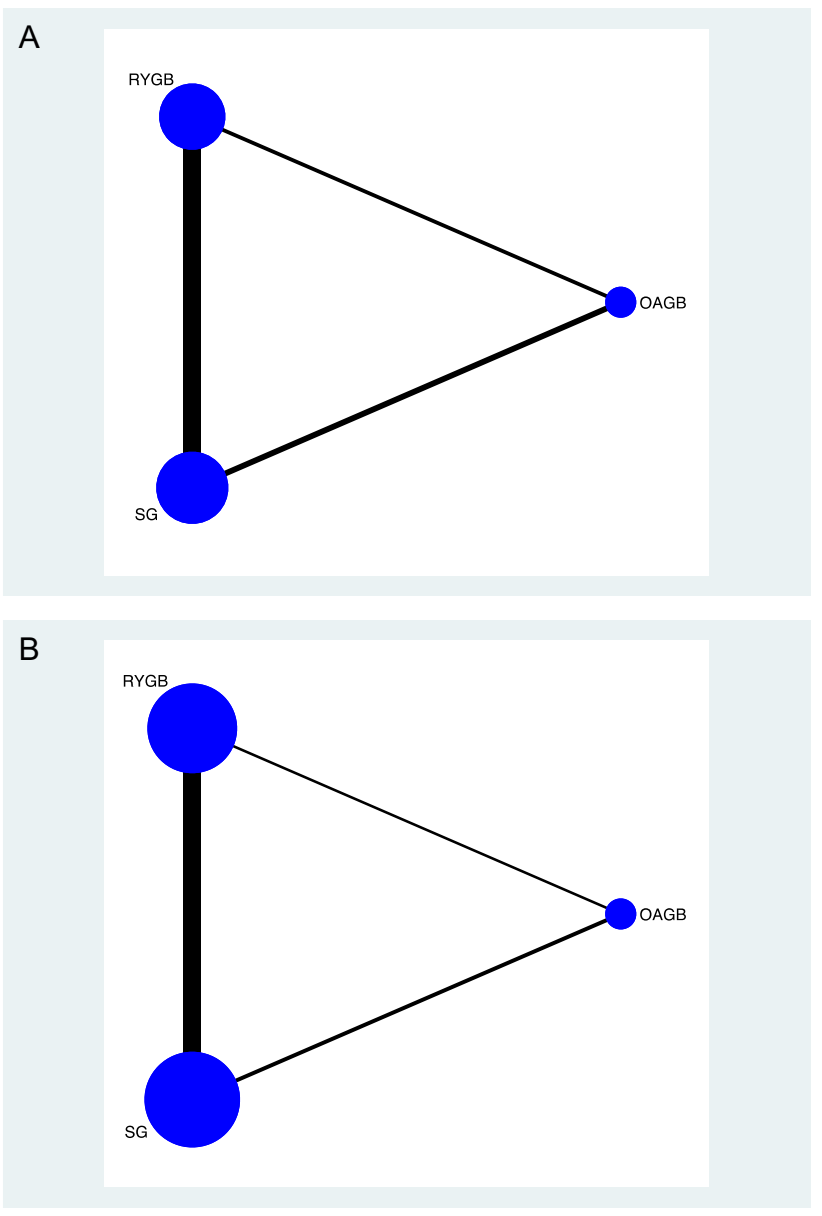

Fig. 3 Network plot for eligible comparisons in perioperative strategies for a excess weight loss (1 year) and b T2DM remission. The size of the nodes is proportional to the number of patients $(n)$ randomised to receive the treatment. The width of the lines is proportional to the number of trials comparing the connected treatment strategies

remission $[36-38,41,43-45,47,48,50,52,53,55,58$, 60] (Table 3). Ten RCTS compared RYGB with SG, 2 RCTs compared RYGB v OAGB and 2 RCTS compared SG with OAGB. In standard meta-analysis, RYGB was $20 \%$ more likely to result in remission of type 2 diabetes compared to SG. There was no difference in T2DM remission between OAGB and either RYGB or SG. On network meta-analysis, both RYGB and OAGB had increased postoperative remission of type 2 diabetes. There were no differences between RYGB and OAGB for remission of type 2 diabetes. In the NMA, there was no inconsistency seen between the direct and network effect sizes $\left(\chi^{2}=7.99,3\right.$ d.f., $P=0.0397)$. No evidence of statistical heterogeneity was seen in the network $\left(\tau^{2}<0.001\right)$.

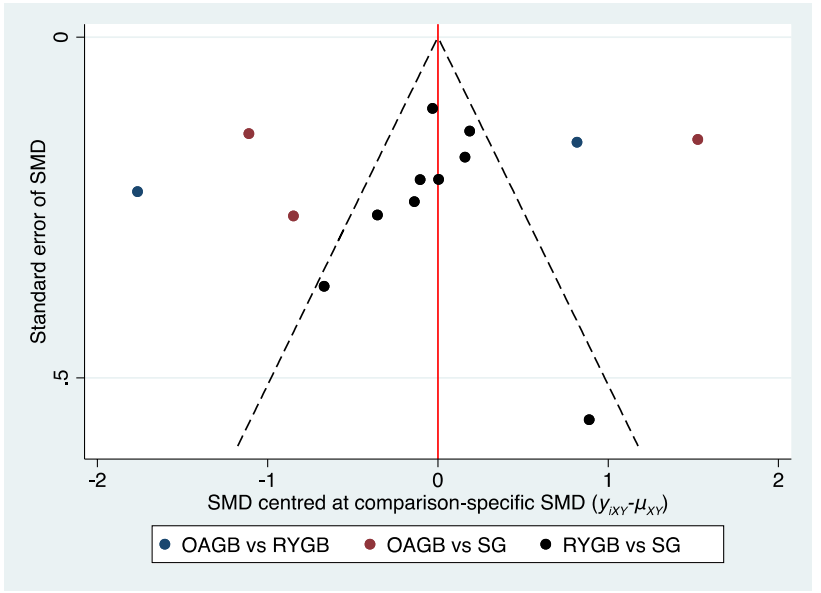

Fig. 4 Comparison-adjusted funnel plot for the outcome of excess weight loss (1 year). Comparisons are made comparing newer treatments against older treatments (RYGB being the oldest, followed by SG and then OAGB). The horizontal axis is the study-specific effect sizes centred to the respective comparison-specific pooled effect size, and the vertical axis is the inverted standard error of the effect sizes as used in a standard funnel plot. The solid red line represents the null hypothesis that the study-specific effect sizes do not differ from the respective comparison-specific pooled treatment effect estimates. Symmetrical distribution of this funnel plot suggests there is no small-study effect in this comparison. SMD standardised mean difference

\section{Perioperative Complications}

Thirteen RCTs including 1593 randomised patients were included in the comparison of perioperative complications [35, 39-44, 46-48, 52, 55-58] (Table 4). Nine RCTs compared RYGB with SG, 2 RCTs compared RYGB v OAGB and 2 RCTS compared SG with OAGB. In standard meta-analysis, RYGB was more likely to result in perioperative complications compared to both SG and OAGB. There was no difference in perioperative complications between OAGB and SG. Similarly, on network meta-analysis, RYGB had increased perioperative complications compared to both SG and OAGB, and there were no differences between SG and OAGB for the development of perioperative complications. In the NMA, there was no inconsistency seen between the direct and network effect sizes $\left(\chi^{2}=1.92,3\right.$ d.f., $\left.P=0.0340\right)$. No evidence of statistical heterogeneity was seen in the network $\left(\tau^{2}<0.001\right)$.

\section{Cluster Plots of Benefits and Risk}

SUCRA clustered ranking plots are shown in Fig. 5. For all years, OAGB consistently ranked highest in terms of maximising EWL and T2DM remission whilst reducing the risk of perioperative complications. 
Table 2 Standard pairwise meta-analysis and network meta-analysis of excess weight loss following metabolic surgery

\begin{tabular}{|c|c|c|c|c|c|c|c|}
\hline Time point & Intervention & Comparator & $\begin{array}{l}\text { No. of direct com- } \\
\text { parison studies }\end{array}$ & $\begin{array}{l}\text { Pairwise meta- } \\
\text { analysis (SMD)* }\end{array}$ & $\begin{array}{l}\text { Between-study } \\
\text { variance (MA) }\end{array}$ & $\begin{array}{l}\text { Network meta- } \\
\text { analysis (SMD)* }\end{array}$ & $\begin{array}{l}\text { Between-study } \\
\text { variance (NMA) }\end{array}$ \\
\hline \multirow[t]{3}{*}{1 year } & RYGB & SG & 9 & $\begin{array}{l}0.06 \\
(-0.09,0.22)\end{array}$ & 0.200 & $\begin{array}{l}1.67 \\
(-0.89,4.24)\end{array}$ & 0.202 \\
\hline & RYGB & OAGB & 2 & $\begin{array}{l}1.76 \\
(-0.77,0.77)\end{array}$ & 0.210 & $\begin{array}{l}4.35 \\
(1.30,7.40)\end{array}$ & 0.005 \\
\hline & SG & OAGB & 2 & $\begin{array}{l}1.21 \\
(-0.64,3.13)\end{array}$ & 0.233 & $\begin{array}{l}2.68 \\
(0.16,5.21)\end{array}$ & 0.037 \\
\hline \multirow[t]{3}{*}{2 years } & RYGB & SG & 5 & $\begin{array}{l}0.05 \\
(-0.024,0.14)\end{array}$ & 0.347 & $\begin{array}{l}1.37 \\
(-2.31,5.06)\end{array}$ & 0.465 \\
\hline & RYGB & OAGB & 3 & $\begin{array}{l}1.00 \\
(-0.63,2.63)\end{array}$ & 0.222 & $\begin{array}{l}3.84 \\
(0.47,7.22)\end{array}$ & 0.026 \\
\hline & SG & OAGB & 2 & $\begin{array}{l}1.26 \\
(-1.01,3.54)\end{array}$ & 0.309 & $\begin{array}{l}2.47 \\
(-0.85,5.79)\end{array}$ & 0.144 \\
\hline \multirow[t]{3}{*}{$3-5$ years } & RYGB & SG & 7 & $\begin{array}{l}-0.28 \\
(-0.49,-0.07)\end{array}$ & 0.024 & $\begin{array}{l}-7.19 \\
(-10.88,-3.51)\end{array}$ & $<0.001$ \\
\hline & RYGB & OAGB & 1 & N/A & - & $\begin{array}{l}0.85 \\
(-3.24,4.93)\end{array}$ & 0.685 \\
\hline & SG & OAGB & 2 & $\begin{array}{l}1.78 \\
(-1.22,4.78)\end{array}$ & 0.301 & $\begin{array}{l}8.04 \\
(3.98,12.10)\end{array}$ & $<0.001$ \\
\hline
\end{tabular}

*Values in parentheses are $95 \%$ confidence intervals. A standardised mean difference (SMD) greater than 0.00 favours the comparator (more EWL with comparator than with intervention); a SMD of less than 0.00 favours intervention (less EWL with comparator than with intervention).

$M A$ standard pairwise meta-analysis, NMA network meta-analysis.

Table 3 Standard pairwise meta-analysis and network meta-analysis of T2DM remission following metabolic surgery

\begin{tabular}{|c|c|c|c|c|c|c|}
\hline Intervention & Comparator & $\begin{array}{l}\text { No. of direct com- } \\
\text { parison studies }\end{array}$ & $\begin{array}{l}\text { Pairwise meta- } \\
\text { analysis (RR)* }\end{array}$ & $\begin{array}{l}\text { Between-study vari- } \\
\text { ance (MA) }\end{array}$ & $\begin{array}{l}\text { Network meta- } \\
\text { analysis (RR)* }\end{array}$ & $\begin{array}{l}\text { Between- } \\
\text { study variance } \\
\text { (NMA) }\end{array}$ \\
\hline RYGB & SG & 11 & $\begin{array}{l}0.62 \\
(0.29,0.95)\end{array}$ & 0.011 & $\begin{array}{l}0.55 \\
(0.34,0.90)\end{array}$ & 0.016 \\
\hline RYGB & OAGB & 2 & $\begin{array}{l}1.05 \\
(0.95,1.15)\end{array}$ & 0.411 & $\begin{array}{l}1.82 \\
(0.70,4.71)\end{array}$ & 0.215 \\
\hline SG & OAGB & 2 & $\begin{array}{l}1.45 \\
(0.90,2.44)\end{array}$ & 0.101 & $\begin{array}{l}3.31 \\
(1.33,8.23)\end{array}$ & 0.010 \\
\hline
\end{tabular}

*Values in parentheses are 95\% confidence intervals. A risk ratio (RR) greater than 1.00 favours the comparator (more desirable events with comparator than with intervention); a RR of less than 1.00 favours intervention (fewer desirable events with comparator than with intervention).

$M A$ standard pairwise meta-analysis, NMA network meta-analysis.

Table 4 Standard pairwise meta-analysis and network meta-analysis of perioperative complications following metabolic surgery

\begin{tabular}{llllll}
\hline Intervention & Comparator & $\begin{array}{l}\text { No. of direct com- } \\
\text { parison studies }\end{array}$ & $\begin{array}{l}\text { Pairwise meta- } \\
\text { analysis (RR)* }\end{array}$ & $\begin{array}{l}\text { Between-study vari- } \\
\text { ance (MA) }\end{array}$ & $\begin{array}{l}\text { Network meta- } \\
\text { analysis (RR)* }\end{array}$ \\
\hline RYGB & SG & 9 & $\begin{array}{l}\text { Between- } \\
\text { study variance } \\
\text { (NMA) }\end{array}$ \\
RYGB & OAGB & 1 & $\begin{array}{l}0.61 \\
(0.32,0.92)\end{array}$ & 0.033 & $\begin{array}{l}0.53 \\
(0.38-0.75)\end{array}$ \\
SG & OAGB & 2 & 0.57 & 0.210 & 0.42 \\
$(0.24-0.72)$ & 0.78 \\
$(0.47,1.32)$
\end{tabular}

*Values in parentheses are $95 \%$ confidence intervals. A risk ratio (RR) greater than 1.00 favours the intervention (fewer events with intervention than with comparator); a RR of less than 1.00 favours comparator (fewer undesirable events with comparator than with intervention).

$M A$ standard pairwise meta-analysis, $N M A$ network meta-analysis. 
A Excess weight loss (at 1 year)

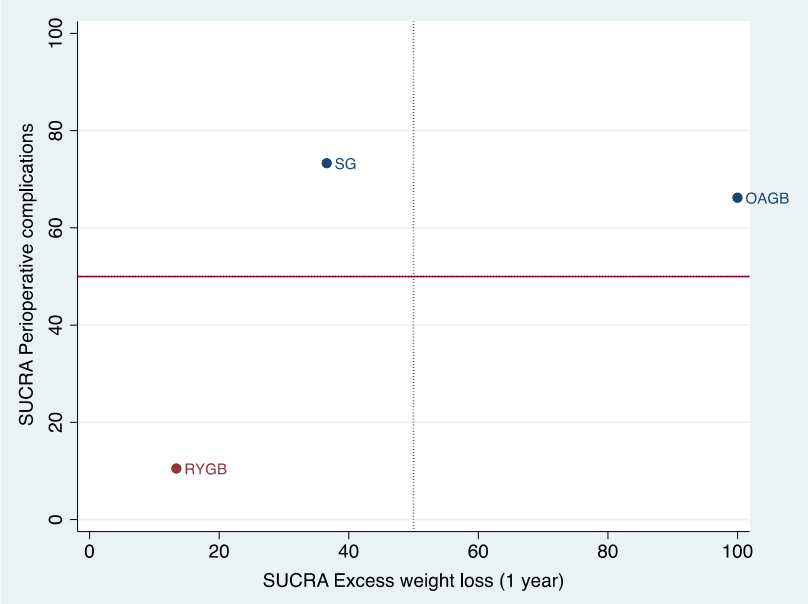

C Excess weight loss (at 3-5 years)

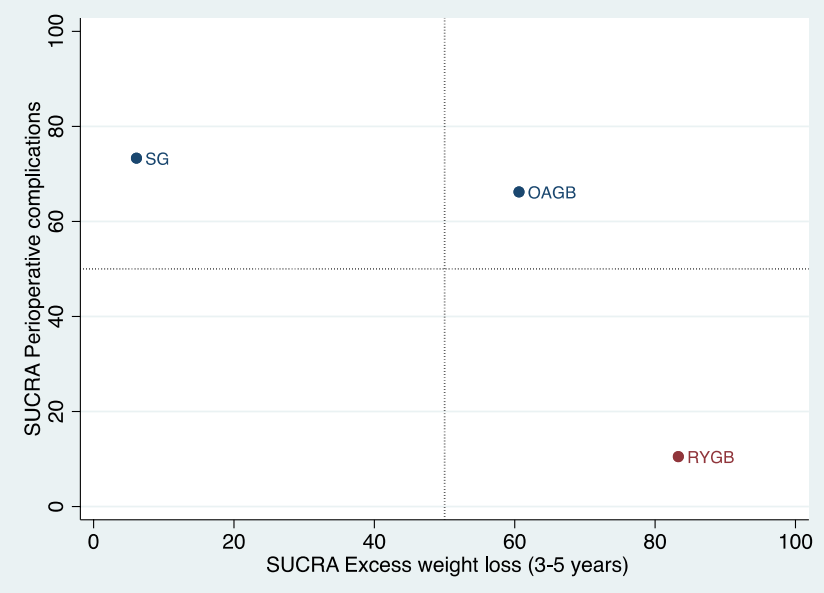

Fig. 5 Clustered ranking plots of the metabolic surgery network based on cluster analysis of SUCRA values for two sets of different outcomes: i) excess weight loss (EWL) and perioperative complica-

\section{Discussion}

This network meta-analysis has uniquely summarised all RCTs to date comparing the most commonly performed current metabolic surgical procedures, i.e. RYGB, SG and OAGB. The EWL demonstrated minor differences at the 1-year and 2-year time points, but no differences between procedures at longer term follow-up. Remission of type 2 diabetes was more likely to occur with either RYGB or OAGB when compared to SG. The risk of perioperative complications was higher with RYGB when compared to either SG or OAGB. When the potential benefits of EWL and T2DM remission were considered against the risk of perioperative complications, OAGB appears to offer the optimal balance of these factors across all time points. The findings from this NMA suggest that OAGB offers comparable metabolic control through weight loss and
B Excess weight loss (at 2 years)

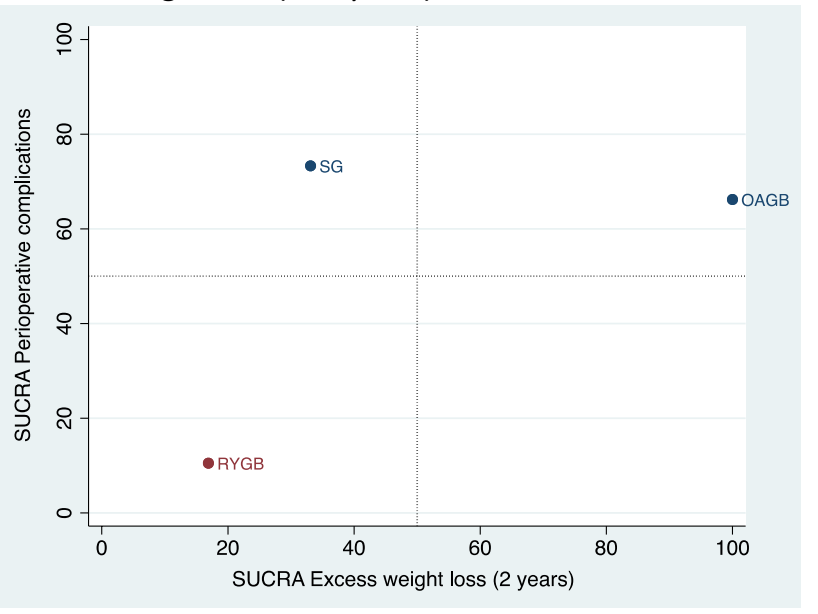

D T2DM remission (any timepoint)

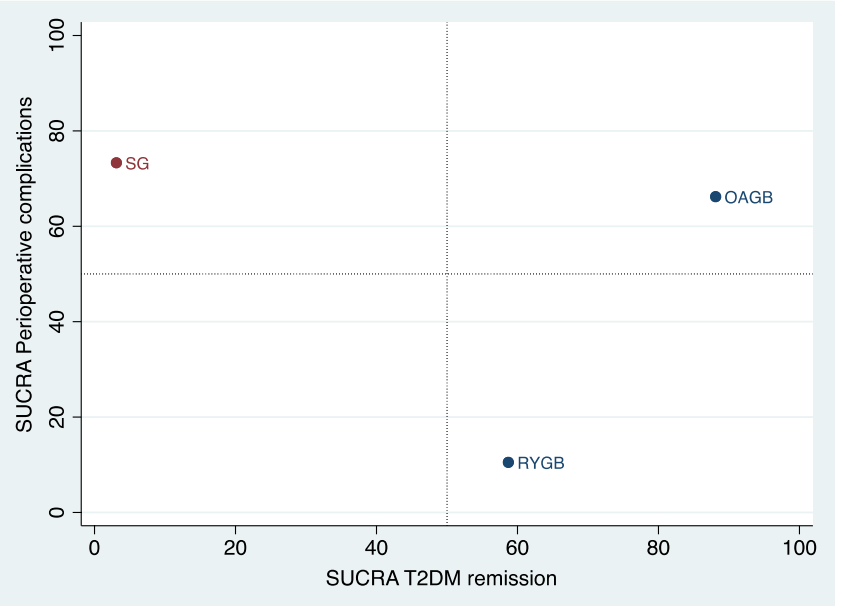

tions, and ii) type 2 diabetes (T2DM) remission and perioperative complications. Treatments lying in the upper right corner are more effective and carry less risk than the other treatments

T2DM remission to RYGB and SG whilst minimising perioperative complications. Therefore, OAGB should be considered more strongly as a primary metabolic surgical procedure.

Previously published NMA studies in bariatric surgery have reported conflicting results. Park and colleagues [61] found that RYGB, SG or biliopancreatic diversion procedures, but not OAGB, produced equally good short and longterm T2DM remission. They considered a larger number of randomised interventions in the formation of their network; however, not all would be currently considered reasonable metabolic surgery interventions. The premise of NMA is that the included interventions could be 'jointly randomisable' as though offered in a contemporary RCT [62]. Banded bypass, gastric plication and even adjustable gastric banding are not considered standard practice in many parts of the world. In comparison, the current NMA compares the three 
most commonly performed metabolic surgical procedures worldwide.

According to the most recent IFSO registry report, the three most commonly performed procedures are RYGB, SG and OAGB [18]. IFSO endorses all of these procedures for use as metabolic surgical interventions $[60,63$, 64]. RYGB is the acknowledged gold standard metabolic surgical intervention and has the most direct and indirect evidence supporting its use in this NMA. SG demonstrates excellent weight loss and metabolic control but is shown to be slightly inferior to RYGB when considering treatment of T2DM alone. This reason for the differences between bypass procedures and SG in metabolic control may be due to improved hepatic insulin sensitivity [38]. This is reflected in the increased use of RYGB, but also OAGB, compared to $\mathrm{SG}$ when considering metabolic surgery for T2DM in a recent IFSO report [65]. This NMA indicates that OAGB may offer acceptable weight loss and glycaemic control as a metabolic surgical procedure. However, there are concerns in the literature about the use of OAGB particularly with regard to risks of protein-calorie malnutrition and upper gastrointestinal malignancies related to bile reflux [66]. Whilst the rates of malnutrition reported in large multinational cohorts are low [67], a recent IFSO survey on OAGB recognised the potential serious risks of malnutrition, liver failure and bile reflux that may require surgical correction after OAGB [68]. Specifically relating to biliary reflux, there are case reports of biliary reflux associated cancers in the context of OAGB [69]. Whilst the overall risk of this complication is unclear, this should merit further investigation. There has been some debate that the limb length in OAGB may influence malnutrition [70], but there remains a lack of consensus on the optimal limb lengths in OAGB [68]. In the current NMA, the included trials had limb lengths from 200 to $350 \mathrm{~cm}[43,47]$. Some groups have found that reducing the limb length to $150 \mathrm{~cm}$ may obviate the risks of malnutrition [71], without a significant effect on the weight loss outcomes, whereas other groups have reported tailoring the limb length to one-third of the total bowel length offers the optimal metabolic outcome [72]. The latter approach may however be associated with higher risk of bowel injuries.

This NMA has identified comparative efficacies of RYGB, SG and OAGB to effect weight loss and induce remission of T2DM. Other studies have used data points in order to define which metabolic surgical procedures should be preferred for different indications. Aminian and colleagues, using data from 654 patients with diabetes who underwent RYGB or $\mathrm{SG}$ at the Cleveland Clinic, produced the Individualised Metabolic Surgical Score (IMSS) [73]. The IMSS incorporates the duration of T2DM, the number of preoperative antidiabetic medications, preoperative insulin use and glycated haemoglobin percentage (HbA1c (\%)) to score patients into mild, moderate or severe categories. Patients with severe
IMSS are proposed by the authors to undergo RYGB as it produces higher remission in this category on their validation set at a Spanish bariatric centre. No patients undergoing OAGB were included in that study, and there was minimal consideration of perioperative complications. Chiapetta and colleagues using data from the German register for obesity and metabolic surgery evaluated the risk of perioperative complications in patients with varying degree of obesityrelated comorbidity using the Edmonton Obesity Staging System [74]. They found that patients with EOSS scores $>2$ had a higher risk of morbidity which was reduced when either SG or OAGB was employed as the metabolic surgical procedure. The authors propose SG or OAGB is used to minimise risk in patients with severe obesity-related comorbidity. However, that study did not evaluate weight loss or T2DM remission outcomes. The current NMA extends these findings to suggest OAGB should be considered alongside RYGB and SG as an option for metabolic surgery in a patient presenting for treatment of obesity or obesity and T2DM.

This study has shown that both bypass procedures offer comparable weight loss and control of T2DM. The mechanism of action of RYGB is comparatively well characterised [75]. The pathways are likely to be multifactorial including changes in eating behaviour, humoral mediated through glucagon-like peptide 1 (GLP-1), gastroinhibitory peptide, glucagon and ghrelin signalling, bile acid kinetics and microbiome-associated effects. Conversely, the mechanism by which OAGB offers metabolic control comparable to RYGB remains to be established. Preliminary work indicates the mechanism of action of OAGB and RYGB may be similar [76]. Additional work in the experimental animal indicates the mechanism of metabolic improvement may be mediated by GLP-1 and bile acid pathways [77]. Overall, more work is required to characterise the mechanistic effect of OAGB in the human population.

Despite comprehensive reporting of all RCTS comparing RYGB, SG and OAGB, there are some limitations to this study that require consideration. By restricting the analysis to the three most commonly performed metabolic surgery procedures worldwide, some comparisons between techniques have limited direct comparative evidence-in particular, there are only two comparative trials of OAGB and RYGB at 1 and 2-year follow-up and only one at 3-5-year follow-up. Whilst this provides limited direct evidence, the strength of network meta-analysis is that the statistical technique permits use of indirect evidence to increase the precision of the treatment effect estimates. Indeed, by demonstrating consistency between direct and indirect treatment effects at all time points, this NMA analysis has shown the benefit of expanding the trial network beyond direct meta-analysis to maximise use of the randomised trial evidence in the area. However, more direct trials of OAGB and both RYGB and SG are needed to improve our understanding of their respective treatment effects. Whilst 
this study has been able to make a comprehensive comparison of trialist-defined complications, there is limited standardisation of reporting and grading of complications which precludes a more in-depth assessment. By including only RCTs, this NMA has limited reporting of longer term data. This may mean that some aspects of outcomes following these particular metabolic procedures may not have been captured, such as weight regain and the need for revisional procedures. Both RYGB and OAGB are associated with a long-term risk of the development of internal hernias and chronic abdominal pain. SG is associated with a long-term risk of GERD [78], and there are reports of long-term gastro-oesophageal and bile reflux following OAGB $[64,69]$. By comparison with other NMAs, this current study assessed fewer procedural types, and therefore the confidence interval estimates are wider as fewer patients overall are included. However, the strength of this NMA lies in the fact that it meets the requirement of joint randomisability of included interventions [62], which other reviews do not by including procedures that are either not practiced by contemporary surgeons or not endorsed by major societies.

In summary, the current NMA has provided a synthesis of the evidence on the three most commonly used metabolic surgical procedures at present. RYGB is associated with the highest rate of perioperative complications. Weight loss at 1 and 2 years appeared greatest with OAGB, but at longer follow-up the differences were less certain. Remission of T2DM was more common with RYGB or OAGB compared to SG. This study has confirmed that RYGB, OAGB or SG should be considered as primary metabolic surgical procedures, and individualised approaches would be reasonable with any of these operations.

Author Contribution ACC, AA, AF \& KM were involved in research design. ACC, AA and AF were involved in data acquisition. ACC, $\mathrm{AA}, \mathrm{AF} \& \mathrm{KM}$ were involved in data interpretation, production of the manuscript and critical revisions. All authors approved the final submitted manuscript.

\section{Declarations}

Ethics Approval This study was a systematic review of published research; therefore, no ethical or governance approvals were required.

Conflict of Interest ACC, AA and AF have no conflicts of interest or financial ties to disclose. KM has received honoraria from Ethicon, Medtronic, Olympus, Gore and various NHS Trusts for educational and mentoring activities related to the OAGB.

\section{References}

1. Sjostrom L, Narbro K, Sjostrom CD, et al. Effects of bariatric surgery on mortality in Swedish obese subjects. N Engl J Med. 2007;357(8):741-52.

2. Gulliford MC, Charlton J, Prevost T, et al. Costs and outcomes of increasing access to bariatric surgery: cohort study and cost-effectiveness analysis using electronic health records. Value Health. 2017;20(1):85-92.

3. Picot J, Jones J, Colquitt JL, et al. The clinical effectiveness and cost-effectiveness of bariatric (weight loss) surgery for obesity: a systematic review and economic evaluation. Health Technol Assess. 2009;13(41):1-190. 215-357

4. Colquitt JL, Pickett K, Loveman E, et al. Surgery for weight loss in adults. Cochrane Database Syst Rev. 2014;8(8):CD003641.

5. Dixon JB, le Roux CW, Rubino F, et al. Bariatric surgery for type 2 diabetes. Lancet. 2012;379(9833):2300-11.

6. Pareek M, Schauer PR, Kaplan LM, et al. Metabolic surgery: weight loss, diabetes, and beyond. J Am Coll Cardiol. 2018;71(6):670-87.

7. Mackenzie H, Markar SR, Askari A, et al. Obesity surgery and risk of cancer. Br J Surg. 2018;105(12):1650-7.

8. Stefanova I, Currie AC, Newton RC, et al. A meta-analysis of the impact of bariatric surgery on back pain. Obes Surg. 2020;30(8):3201-7.

9. Rubino F, Nathan DM, Eckel RH, et al. Metabolic surgery in the treatment algorithm for type 2 diabetes: a joint statement by International Diabetes Organizations. Diabetes Care. 2016;39(6):861-77.

10. Docherty AB, Harrison EM, Green CA, et al. Features of 20133 UK patients in hospital with COVID-19 using the ISARIC WHO Clinical Characterisation Protocol: prospective observational cohort study. BMJ. 2020;369:m1985.

11. Cariou B, Hadjadj S, Wargny M, et al. Phenotypic characteristics and prognosis of inpatients with COVID-19 and diabetes: the CORONADO study. Diabetologia. 2020;29

12. Rubino F, Cohen RV, Mingrone G, et al. Bariatric and metabolic surgery during and after the COVID-19 pandemic: DSS recommendations for management of surgical candidates and postoperative patients and prioritisation of access to surgery. Lancet Diabetes Endocrinol. 2020;8(7):640-8.

13. Hussain A, Mahawar K, El-Hasani S. The impact of COVID19 pandemic on obesity and bariatric surgery. Obes Surg. 2020;30(8):3222-3.

14. Currie AC, Askari A, Newton RC, et al. Regional variation in unmet need for metabolic surgery in England: a retrospective, multicohort analysis. Obes Surg. 2021;31(1):439-44.

15. Dixon JB. Regional differences in the coverage and uptake of bariatric-metabolic surgery: a focus on type 2 diabetes. Surg Obes Relat Dis. 2016;12(6):1171-7.

16. Welbourn R, Dixon J, Barth JH, et al. NICE-accredited commissioning guidance for weight assessment and management clinics: a model for a specialist multidisciplinary team approach for people with severe obesity. Obes Surg. 2016;26(3):649-59.

17. Welbourn R, Hopkins J, Dixon JB, et al. Commissioning guidance for weight assessment and management in adults and children with severe complex obesity. Obes Rev. 2018;19(1):14-27.

18. Welbourn R, Hollyman M, Kinsman R, et al. Bariatric surgery worldwide: baseline demographic description and one-year outcomes from the Fourth IFSO Global Registry Report 2018. Obes Surg. 2019;29(3):782-95.

19. Borgeraas H, Hofso D, Hertel JK, et al. Comparison of the effect of Roux-en-Y gastric bypass and sleeve gastrectomy on remission of type 2 diabetes: a systematic review and meta-analysis of randomized controlled trials. Obes Rev. 2020;21(6):e13011. 
20. Magouliotis DE, Tasiopoulou VS, Tzovaras G. One anastomosis gastric bypass versus Roux-en-Y gastric bypass for morbid obesity: an updated meta-analysis. Obes Surg. 2019;29(9):2721-30.

21. Jia D, Tan H, Faramand A, et al. One anastomosis gastric bypass versus Roux-en-Y gastric bypass for obesity: a systematic review and meta-analysis of randomized clinical trials. Obes Surg. 2020;30(4):1211-8.

22. Madadi F, Jawad R, Mousati I, et al. Remission of type 2 diabetes and sleeve gastrectomy in morbid obesity: a comparative systematic review and meta-analysis. Obes Surg. 2019;29(12):4066-76.

23. Angrisani L, Santonicola A, Iovino P, et al. IFSO worldwide survey 2016: primary, endoluminal, and revisional procedures. Obes Surg. 2018;28(12):3783-94. Epub 2018/08/20

24. Moher D, Liberati A, Tetzlaff J, et al. Preferred reporting items for systematic reviews and meta-analyses: the PRISMA statement. Ann Intern Med. 2009;151(4):264-9. W64

25. Hutton B, Salanti G, Caldwell DM, et al. The PRISMA extension statement for reporting of systematic reviews incorporating network meta-analyses of health care interventions: checklist and explanations. Ann Intern Med. 2015;162(11):777-84.

26. Higgins JPT, Altman DG, Gøtzsche PC, et al. The Cochrane collaboration's tool for assessing risk of bias in randomised trials. 2011 2011-10-18 10:55:48.

27. Sidik K, Jonkman JN. A comparison of heterogeneity variance estimators in combining results of studies. Stat Med. 2007;26(9):1964-81.

28. Raudenbush SW. Analyzing effect sizes: random effects models. In: Cooper H, Hedges LV, Valentine JC, editors. The handbook of research synthesis and meta-analysis. 2nd ed. New York: Russell Sage Foundation; 2009. p. 295-315.

29. Jackson D, Barrett JK, Rice S, et al. A design-by-treatment interaction model for network meta-analysis with random inconsistency effects. Stat Med. 2014;33(21):3639-54.

30. Higgins JPT, Jackson D, Barrett JK, et al. Consistency and inconsistency in network meta-analysis: concepts and models for multiarm studies. Res Synth Methods. 2012;3(2):98-110.

31. White IR, Barrett JK, Jackson D, et al. Consistency and inconsistency in network meta-analysis: model estimation using multivariate meta-regression. Res Synth Methods. 2012;3(2):111-25.

32. Chaimani A, Higgins JPT, Mavridis D, et al. Graphical tools for network meta-analysis in STATA. PLoS One. 2013;8(10):e76654.

33. White IR. Multivariate random-effects meta-regression: updates to mvmeta. Stata J. 2011;11(2):255-70.

34. White IR. Network meta-analysis. Stata J. 2015;15(4):951-85.

35. Biter LU, Buuren MMA, Mannaerts GHH, et al. Quality of life 1 year after laparoscopic sleeve gastrectomy versus laparoscopic Roux-en-Y gastric bypass: a randomized controlled trial focusing on gastroesophageal reflux disease. Obes Surg. 2017;27

36. Casajoana A, Pujol J, Garcia A, et al. Predictive value of gut peptides in T2D remission: randomized controlled trial comparing metabolic gastric bypass, sleeve gastrectomy and greater curvature plication. Obes Surg. 2017;27

37. Gras-Miralles B, Haya JR, Moros JM, et al. Caloric intake capacity as measured by a standard nutrient drink test helps to predict weight loss after bariatric surgery. Obes Surg. 2014;24

38. Hofso D, Fatima F, Borgeraas H, et al. Gastric bypass versus sleeve gastrectomy in patients with type 2 diabetes (Oseberg): a single-centre, triple-blind, randomised controlled trial. Lancet Diabetes Endocrinol. 2019;7(12):912-24.

39. Ignat M, Vix M, Imad I, et al. Randomized trial of Roux-en-Y gastric bypass versus sleeve gastrectomy in achieving excess weight loss. Br J Surg. 2017;104

40. Karamanakos SN, Vagenas K, Kalfarentzos F, et al. Weight loss, appetite suppression, and changes in fasting and postprandial ghrelin and peptide-YY levels after Roux-en-Y gastric bypass and sleeve gastrectomy: a prospective, double blind study. Ann Surg. 2008;247

41. Kehagias I, Karamanakos SN, Argentou M, et al. Randomized clinical trial of laparoscopic Roux-en-Y gastric bypass versus laparoscopic sleeve gastrectomy for the management of patients with BMI $<50 \mathrm{~kg} / \mathrm{m} 2$. Obes Surg. 2011;21

42. Lee WJ, Chong K, Ser KH, et al. Gastric bypass vs sleeve gastrectomy for type 2 diabetes mellitus: a randomized controlled trial. Arch Surg. 2011;146

43. Lee WJ, Yu PJ, Wang W, et al. Laparoscopic Roux-en-Y versus mini-gastric bypass for the treatment of morbid obesity: a prospective randomized controlled clinical trial. Ann Surg. 2005;242

44. Paluszkiewicz R, Kalinowski P, Wroblewski T, et al. Prospective randomized clinical trial of laparoscopic sleeve gastrectomy versus open Roux-en-Y gastric bypass for the management of patients with morbid obesity. Wideochir Inne Tech Maloinwazyjne. 2012;7

45. Peterli R, Wolnerhanssen BK, Peters T, et al. Effect of laparoscopic sleeve gastrectomy vs laparoscopic Roux-en-Y gastric bypass on weight loss in patients with morbid obesity: the SMBOSS randomized clinical trial. JAMA. 2018;319

46. Peterli R, Wolnerhanssen BK, Vetter D, et al. Laparoscopic sleeve gastrectomy versus Roux-Y-gastric bypass for morbid obesity3 -year outcomes of the prospective randomized Swiss multicenter bypass or sleeve study (SM-BOSS). Ann Surg. 2017;265

47. Robert M, Espalieu P, Pelascini E, et al. Efficacy and safety of one anastomosis gastric bypass versus Roux-en-Y gastric bypass for obesity (YOMEGA): a multicentre, randomised, open-label, non-inferiority trial. Lancet. 2019;393(10178):1299-309.

48. Salminen P, Helmio M, Ovaska J, et al. Effect of laparoscopic sleeve gastrectomy vs laparoscopic Roux-en-Y gastric bypass on weight loss at 5 years among patients with morbid obesity: the SLEEVEPASS randomized clinical trial. JAMA. 2018;319

49. Schauer PR, Bhatt DL, Kirwan JP, et al. Bariatric surgery versus intensive medical therapy for diabetes - 5-year outcomes. N Engl J Med. 2017;376(7):641-51.

50. Schauer PR, Bhatt DL, Kirwan JP, et al. Bariatric surgery versus intensive medical therapy for diabetes-3-year outcomes. N Engl J Med. 2014;370(21):2002-13.

51. Schauer PR, Kashyap SR, Wolski K, et al. Bariatric surgery versus intensive medical therapy in obese patients with diabetes. N Engl J Med. 2012;366(17):1567-76.

52. Seetharamaiah S, Tantia O, Goyal G, et al. LSG vs OAGB-1 year follow-up data-a randomized control trial. Obes Surg. 2017;27

53. Tang Q, Sun Z, Zhang N, et al. Cost-effectiveness of bariatric surgery for type 2 diabetes mellitus: a randomized controlled trial in China. Medicine (Baltimore). 2016;95(20):e3522.

54. Vix M, Diana M, Liu KH, et al. Evolution of glycolipid profile after sleeve gastrectomy vs. Roux-en-Y gastric bypass: results of a prospective randomized clinical trial. Obes Surg. 2013;23(5):613-21.

55. Yang J, Wang C, Cao G, et al. Long-term effects of laparoscopic sleeve gastrectomy versus roux-en-Y gastric bypass for the treatment of Chinese type 2 diabetes mellitus patients with body mass index 28-35 kg/m(2). BMC Surg. 2015;15

56. Zhang $\mathrm{Y}$, Zhao $\mathrm{H}, \mathrm{Cao} \mathrm{Z}$, et al. A randomized clinical trial of laparoscopic Roux-en-Y gastric bypass and sleeve gastrectomy for the treatment of morbid obesity in China: a 5-year outcome. Obes Surg. 2014;24

57. Jain M, Tantia O, Goyal G, et al. LSG vs MGB-OAGB: 5-year follow-up data and comparative outcome of the two procedures over long term-results of a randomised control trial. Obes Surg. 2020;

58. Level L, Rojas A, Pinango S, et al. One anastomosis gastric bypass vs. Roux-en-Y gastric bypass: a 5-year follow-up prospective randomized trial. Langenbeck's Arch Surg. 2020; 
59. Shivakumar S, Tantia O, Goyal G, et al. LSG vs MGB-OAGB-3 year follow-up data: a randomised control trial. Obes Surg. 2018;28(9):2820-8.

60. De Luca M, Angrisani L, Himpens J, et al. Indications for surgery for obesity and weight-related diseases: position statements from the International Federation for the Surgery of Obesity and Metabolic Disorders (IFSO). Obes Surg. 2016;26(8):1659-96.

61. Park CH, Nam SJ, Choi HS, et al. Comparative efficacy of bariatric surgery in the treatment of morbid obesity and diabetes mellitus: a systematic review and network meta-analysis. Obes Surg. 2019;29(7):2180-90.

62. Riley RD, Jackson D, Salanti G, et al. Multivariate and network meta-analysis of multiple outcomes and multiple treatments: rationale, concepts, and examples. BMJ. 2017;358:j3932.

63. Ramos AC, Chevallier JM, Mahawar K, et al. IFSO (International Federation for Surgery of Obesity and Metabolic Disorders) consensus conference statement on one-anastomosis gastric bypass (OAGB-MGB): results of a modified Delphi study. Obes Surg. 2020;30(5):1625-34.

64. De Luca M, Tie T, Ooi G, et al. Mini gastric bypass-one anastomosis gastric bypass (MGB-OAGB)-IFSO position statement. Obes Surg. 2018;28(5):1188-206.

65. Welbourn R, Hollyman M, Kinsman R, et al. Bariatric-metabolic surgery utilisation in patients with and without diabetes: data from the IFSO Global Registry 2015-2018. Obes Surg. 2021;

66. Parikh M, Eisenberg D, Johnson J, et al. American Society for M, Bariatric surgery clinical issues C. American Society for Metabolic and Bariatric Surgery review of the literature on one-anastomosis gastric bypass. Surg Obes Relat Dis. 2018;14(8):1088-92.

67. Hussain A, Van den Bossche M, Kerrigan DD, et al. Retrospective cohort study of 925 OAGB procedures. The UK MGB/OAGB collaborative group. Int J Surg. 2019;69:13-8.

68. Haddad A, Bashir A, Fobi M, et al. The IFSO worldwide one anastomosis gastric bypass survey: techniques and outcomes? Obes Surg. 2021;

69. Rutledge R, Deitel M, Carbajo MA, et al. Commentary: cancer after the OAGB-MGB. Obes Surg. 2020;30(2):755-8.

70. Mahawar KK, Parmar C, Carr WRJ, et al. Impact of biliopancreatic limb length on severe protein-calorie malnutrition requiring revisional surgery after one anastomosis (mini) gastric bypass. J Minimal Access Surg. 2018;14(1):37-43.
71. Boyle M, Mahawar K. One anastomosis gastric bypass performed with a $150-\mathrm{cm}$ biliopancreatic limb delivers weight loss outcomes similar to those with a $200-\mathrm{cm}$ biliopancreatic limb at $18-24$ months. Obes Surg. 2020;30(4):1258-64.

72. Abdallah E, Emile SH, Zakaria M, et al. One-anastomosis gastric bypass (OAGB) with fixed bypass of the proximal two meters versus tailored bypass of the proximal one-third of small bowel: short-term outcomes. Surg Endosc. 2021;

73. Aminian A, Brethauer SA, Andalib A, et al. Individualized metabolic surgery score: procedure selection based on diabetes severity. Ann Surg. 2017;266(4):650-7.

74. Chiappetta S, Stier C, Weiner RA. members of StuDo Q, Viszeralchirurgie/StuDoQ MBEoDGfA-u. The Edmonton Obesity Staging System predicts perioperative complications and procedure choice in obesity and metabolic surgery-a German nationwide register-based cohort study (StuDoQ|MBE). Obes Surg. 2019;29(12):3791-9.

75. Russel SM, Valle V, Spagni G, et al. Physiologic mechanisms of Type II diabetes mellitus remission following bariatric surgery: a meta-analysis and clinical implications. J Gastrointest Surg. 2020;24(3):728-41.

76. Dardzinska JA, Kaska L, Wisniewski P, et al. Fasting and post-prandial peptide YY levels in obese patients before and after mini versus Roux-en-Y gastric bypass. Minerva Chir. 2017;72(1):24-30.

77. Marciniak C, Chavez-Talavera O, Caiazzo R, et al. Characterization of one anastomosis gastric bypass and impact of biliary and common limbs on bile acid and postprandial glucose metabolism in a minipig model. Am J Physiol Endocrinol Metab. 2021;25

78. Yeung KTD, Penney N, Ashrafian L, et al. Does sleeve gastrectomy expose the distal esophagus to severe reflux?: a systematic review and meta-analysis. Ann Surg. 2020;271(2):257-65.

Publisher's Note Springer Nature remains neutral with regard to jurisdictional claims in published maps and institutional affiliations. 